Relations industrielles

Industrial Relations

\title{
National Academy of Arbitrators and the NAA Research and Education Foundation, Labour Arbitration in America
}

\section{Claire Brassard}

Volume 48, numéro 1, 1993

URI : https://id.erudit.org/iderudit/050843ar

DOI : https://doi.org/10.7202/050843ar

Aller au sommaire du numéro

Éditeur(s)

Département des relations industrielles de l'Université Laval

ISSN

0034-379X (imprimé)

1703-8138 (numérique)

Découvrir la revue

Citer ce compte rendu

Brassard, C. (1993). Compte rendu de [National Academy of Arbitrators and the NAA Research and Education Foundation, Labour Arbitration in America]. Relations industrielles / Industrial Relations, 48(1), 198-201.

https://doi.org/10.7202/050843ar

Tous droits réservés @ Département des relations industrielles de l'Université Laval, 1993
Ce document est protégé par la loi sur le droit d'auteur. L’utilisation des services d'Érudit (y compris la reproduction) est assujettie à sa politique d'utilisation que vous pouvez consulter en ligne.

https://apropos.erudit.org/fr/usagers/politique-dutilisation/ 
movement" and appears to doubt the robustness of the existing leadership to tackle recessionary pressures. He also doubts its ability to transcend pure and simple unionism. This throws the spotlight upon unions and politics. Unfortunately, the relevant chapter by Delaney and Masters does not operate on a complementary level. We do learn that unions invariably involve themselves in politics to influence the legislative agenda, favourably towards union objectives, meaning their security and the continuation of collective bargaining. At this level, Delaney and Masters provide an excellent service in bringing together the existing literature. However, it might have been more useful to have the authors cast their net in wider terms, both conceptually and comparatively. We really need to know how union movements have responded at the aggregate level to the shifting fortunes of capital. A discussion of corporatism is desperately needed here. Comparative analysis of how British unions (the early versions of the Social Contract) and more recently, the Australian Accords between the ACTU and Labour administrations (now being dismantled) might have enabled us to appraise more usefully the relative strengths and weaknesses of U.S. unions in relation to their political agenda.

In the final segment on the future, articles by Kochan and Weaver and finally Piore, emphasise that unions must construct broader appeals if they are to remain important democratic institutions in American society. The former argue for increasing training and "upskilling" in order to make peripheral workers look more like the "core". Piore argues for further links with the marginalized sections of the labour force within the context of supportive local communities of interest. Hyman, in his commentary, applauds the value of constructing a broader structure of identities for unions but is less than convinced that success is forthcoming. His solution, as an avowed Marxist in the European tradition, is to place greater emphasise on the external origins of workplace antagonisms. He presumably feels that when workers recognise that it is the whims of short-term capital markets which cause their discontent, that internal sectional distinctions may be transcended. Put simply, Hyman is arguing that only when American unions recognise their broader class identity, will their fortunes and those of the working-class in general, improve.

An interesting conclusion indeed to a book typically cast in the traditions of American industrial relations orthodoxy.

Clive H. J. GilsoN

\section{St. Francis Xavier University}

Labour Arbitration in America, research conducted under the auspices of the National Academy of Arbitrators and the NAA Research and Education Foundation, New York, Praeger, 1992, 186 p., ISBN 0-275-94375-5

Cet ouvrage présente les résultats d'une recherche exhaustive menée aux ÉtatsUnis sur le profil des personnes agissant comme arbitre et sur les caractéristiques de leur pratique. La recherche a été dirigée par les professeurs Mario F. Bognanno, directeur du Industrial Relations Center de l'Université du Minnesota et Charles J. Coleman de l'Université Rutgers (Camden). 
Quoique la fonction d'arbitre soit aussi ancienne que les temps bibliques, elle est devenue un métier et elle fait partie intégrante des relations du travail depuis au moins 50 ans aux États-Unis. Les auteurs de l'ouvrage annoncent que leur équipe a produit la recherche la plus complète à ce jour sur le sujet en bénéficiant de la contribution de plusieurs universités américaines. Les objectifs de la recherche étaient de deux ordres. D'abord, colliger les résultats d'une recherche empirique exceptionnellement complète, à partir de données de 1986-87, permettant de tracer l'histoire inconnue de l'arbitrage des relations du travail et le profil des personnes qui en font. Ensuite, écrire un rapport simple, accessible aux non-initiés, présentant les arbitres, leur expérience, leur formation, leurs aspirations, leurs revenus, etc. La recherche a été faite à partir d'un échantillon de 765 personnes qui ont répondu au questionnaire reproduit à la fin du livre.

L'ouvrage comprend sept chapitres. Le premier chapitre présente les objectifs, la nature de la recherche, la méthodologie utilisée et la représentativité de l'échantillon. Systématiquement, dans les résultats, on fait la distinction entre les membres de l'Académie nationale des arbitres (ANA) et les non-membres. Il est utile de savoir que l'ANA est le plus important regroupement d'arbitres aux États-Unis et qu'elle a subventionné la recherche en question. Le second chapitre aborde le portrait des arbitres, à savoir leur niveau de scolarité, leur expérience, leur carrière et leurs revenus, alors que le troisième chapitre présente les mêmes variables, mais observées chez les membres de l'ANA spécifiquement.

Essentiellement, nous apprenons que la majorité des arbitres sont des hommes, de race blanche, âgés et instruits. Il sagit pour la plupart d'entre eux d'un second emploi et ils viennent majoritairement des milieux de l'enseignement et du droit. En somme, toutes choses que nous savions déjà sauf peut-être en ce qui concerne les arbitres qui sont des femmes. On apprend qu'elles sont plus jeunes que leurs collègues masculins, davantage concentrées dans les secteurs public et parapublic et, plus ambitieuses. En 1986, quatre arbitres sur cinq pratiquaient l'arbitrage à temps partiel. Ils entendaient en moyenne 15 cas par année, travaillaient quatre jours par mois en arbitrage et en tiraient près du cinquième de leur revenu annuel. Les arbitres à temps plein, eux, entendaient environ 65 cas par année en 1986. Les revenus et le nombre de dossiers variaient selon que les arbitres étaient membres de l'ANA ou non. Ainsi, la moyenne des honoraires par jour des non-membres était de $374,89 \$$ alors qu'elle était de $435,95 \$$ pour les membres. Il faut ajouter que les membres de l'ANA exigaient des frais d'annulation d'audition de $374,74 \$$ alors que les non-membres en exigaient seulement $180,36 \$$. Finalement, on observait que les arbitres avaient tendance à avoir deux bureaux, pour élargir leur territoire d'intervention mais aussi pour migrer vers le Sud pendant l'hiver.

Le quatrième chapitre analyse la carrière de l'arbitre sur un continuum : les débuts, l'apogée et le déclin. On y fait un examen des besoins de formation pour le développement de carrière et on retrouve à la fin du chapitre un modèle de développement de carrière. L'enquête révèle, on s'en doutait, que les débuts sont longs et difficiles. En revanche, une fois la carrière établie, les arbitres travaillent longtemps, la plupart au-delà de l'âge de 65 ans.

Le chapitre cinq se concentre sur les dossiers d'arbitrage eux-mêmes: leur nombre, leur provenance, les enjeux et la différence de volume de dossiers (case load) entre les arbitres. Les griefs qui ont fait l'objet de décisions arbitrales portent, par ordre 
d'importance en nombre, sur les congédiements, l'absentéisme, la performance au travail, les droits de gestion, l'ancienneté, les pratiques passées. La majeure partie des dossiers proviennent du secteur privé et, par ordre d'importance, des industries manufacturières, des bureaux, des professionnels, des services et du commerce, du transport, etc. Dans le secteur public, les dossiers proviennent, par ordre d'importance, des enseignants, des policiers, des pompiers et du secteur des soins de la santé. Les variables influençant de façon significative le nombre de dossiers traités par les arbitres sont celles reliées à l'intensité de l'activité (temps plein vs temps partiel), l'expérience, la formation en droit et le fait d'être membre de l'ANA.

On aurait pu intituler le chapitre six « Grandeurs et misères de l'arbitrage ». En effet, on y présente les revenus des arbitres, le nombre de décisions rendues, le montant de leurs honoraires, les différentes charges de voyages d'étude et de rédaction. Voici des éléments qui sauront particulièrement intéresser les arbitres canadiens qui pourront se comparer avec leurs collègues américains grâce à une exactitude de données rarement disponible. À cet égard, il faut noter que les données ont été recueillies en 1986 et 1987 et doivent être extrapolées pour donner une idée plus juste de la situation actuelle.

La moyenne annuelle des revenus pour tous les arbitres - temps plein et temps partiel - était de 25579 en 1986 . Toutefois, la moyenne des revenus des arbitres à temps plein et membres de l'ANA, était de 98850 \$ en 1986, contre 44499 \$ pour les non-membres. Il faut mentionner que, pour certains arbitres, il s'agit de revenus s'ajoutant à un revenu de retraite. Enfin, l'arbitre-type pratiquant l'arbitrage à temps partiel en tire un revenu moyen de $15000 \$$.

Le per diem des arbitres est constitué des honoraires et des coûts reliés aux déplacements, à l'audition, aux délibérés, à l'analyse et à la rédaction. Il faut ajouter les coûts d'annulation dont la moyenne était de $216 \$$ en 1986 . La moyenne des jours annulés dans une année représentait 3,4 jours, c'est-à-dire une journée annulée sur huit journées prévues. Il s'agit donc d'un pourcentage plutôt bas (12\%) d'annulations qui n'est pas de nature à bouleverser un agenda de façon importante. La moyenne des revenus pour l'ensemble des dossiers était de $1106 \$$, et la moyenne des revenus des dossiers des membres de l'ANA était de $1238 \$$ tandis que celle des arbitres qui sont des fernmes était de $974 \$$. L'étude signale qu'en fin de compte, les revenus des arbitres se situent en-deçà de ceux d'autres professionnels tels les médecins, les avocats et les ingénieurs.

Enfin, le chapitre sept étudie l'offre et la demande pour les services d'arbitrage par région géographique. On note, pour l'année 1986, plus d'offre que de demande puisque les trois quarts des arbitres auraient souhaité se voir attribuer plus de dossiers. Par ailleurs, il n'est pas étonnant de constater que les arbitres se retrouvent davantage dans les centres urbains, plutôt industrialisés et hautement syndiqués. Les chercheurs soulignent, en effet, la corrélation entre le nombre d'arbitres et le nombre de travailleurs syndiqués par région.

Les auteurs de l'ouvrage avaient d'ambitieux objectifs en voulant tracer un portrait inédit des arbitres et de l'arbitrage par une recherche exhaustive et empirique. En effet, le profil-type de l'arbitre qu'ils ont dégagé de cette recherche n'a rien d'inédit pour les initiés, du moins ils n'apprennent pas beaucoup de choses qu'ils ne savaient ou qu'ils ne soupçonnaient déjà. Le rapport de recherche a cependant la qualité de rassurer les 
initiés en confirmant ce qu'ils savaient par intuition. Soyez désormais avisés que les arbitres américains, à l'instar de leurs collègues canadiens, sont dans l'ensemble des hommes, blancs, âgés, instruits et la plupart du temps retraités. Les femmes arbitres, quant à elles, pénètrent lentement mais sûrement dans le métier.

De cette recherche, il faut aussi souligner la remarquable base de données sur les arbitres et leur pratique arbitrale. Ces données permettront éventuellement de mesurer l'évolution de la pratique de l'arbitrage. Cette étude a enfin permis de dégager des informations très précises auxquelles il est toujours intéressant de se comparer. $\AA$ coup sûr, il s'agit d'un bon document d'archives.

Claire Brassard

Phillips \& Vineberg, Montréal

Trends, Patterns and Impact of Strikes, by Y.R.K. REDDY, Hyderabad, India, Spade Publications, 1990, 114 p., ISBN 81-85491-00-3

The book is about strike trends, their patterns, seasonality, and inter-industry and inter-state propensities for strike activities in India, together with their impact and their policy implications.

In Chapter I, the author has addressed the issues related to definition of industrial conflict, reporting requirements, and computational and measurement problems. In addition, he has developed a portfolio of strike measures for the purpose of empirical analysis of strike activities to follow in the subsequent chapters of the book. Elements included in the portfolio are strike frequency, participation, loss, and duration. Appropriate deflators and possible ratios to standardise these strike measures have also been discussed in this chapter.

Chapter II is a test of Ross-Hartman hypothesis on strike trends, and a reassessment of "withering away of strikes" prediction. This has been done in two ways: (i) by examining the Indian evidence, and (ii) through examinations of comparative data for 14 countries including India. The objective of the comparison was to find relative ranking of the Indian strike situation for the periods 1978-1980 and 1984-1986. Countries used for international comparison include both developed and developing ones. Except for strike frequency, the author found an increasing trend in strike activities in India for the periods examined. In fact, "India ranked first and second among the fourteen countries, in respect of mandays lost per 1,000 employees, during the two periods analysed. Similar was the case in respect of the duration measure. The average duration for India was 25 days in period B as against the lowest of 1.2 days in the case of S. Korea" (p. 32).

In Chapter III, the author has examined causes, duration, and results of strikes during the period from 1951 to 1986 . The analysis indicates that the wage disputes have decreased over the years while strikes in other categories have increased. One of the explanations given for the decline in wage strikes is the setting up of Wage Boards by the government for all the major industries. However, it has been conjectured that disputes in the other categories might have been used increasingly as trade-off for wage bargaining. As to the duration, the author observes a steady decline of shorter duration strikes but an increasing trend in longer duration ones. Explanation for this development 